\title{
THREE-DIMENSIONAL TERMINAL TORIC FLIPS
}

\author{
OSAMU FUJINO, HIROSHI SATO, YUKISHIGE TAKANO, HOKUTO \\ UEHARA
}

\begin{abstract}
We describe three-dimensional terminal toric flips. We obtain the complete local description of three-dimensional terminal toric flips.
\end{abstract}

\section{INTRODUCTION}

The main purpose of this paper is to describe three-dimensional terminal toric flips.

Theorem 1.1 (cf. Theorems 3.1 and 4.1). Let $\varphi: X \rightarrow Y$ be a small proper toric morphism such that $X$ is a three-dimensional toric variety with only terminal singularities. Note that $X$ is not assumed to be $\mathbb{Q}$-factorial. Let $C \simeq \mathbb{P}^{1}$ be an exceptional curve of $\varphi$. Assume that $-K_{X} \cdot C>0$. Then one of the torus invariant points of $X$ on $C$ is non-singular and another one is a terminal quotient singularity. In particular, $X$ is $\mathbb{Q}$-factorial and is not Gorenstein around $C$.

By this result, we obtain the complete local description of threedimensional terminal toric flips (see Theorem 3.1). The first author apologies for the mistake in [F2, Example 4.4.2], where he claims that there exist three-dimensional non- $\mathbb{Q}$-factorial terminal toric flips. However, Theorem 1.1 implies that there are no such flips. This paper is based on the first author's private notes and the third author's master's thesis [T].

We summarize the contents of this paper. In Section 2, we describe three-dimensional terminal toric singularities. The results are well known to the experts. Section 3 gives the complete classification of three-dimensional $\mathbb{Q}$-factorial terminal toric flips. It is a supplement to [KMM and [M, Example-Claim 14-2-5]. In Section 4, we prove that there are no three-dimensional non- $\mathbb{Q}$-factorial terminal toric flips. Theorem 4.1 is the main theorem of this paper. The proof depends on the results in Sections 2 and 3 .

Date: $2008 / 4 / 7$.

2000 Mathematics Subject Classification. Primary 14M25; Secondary 14B05, 14J30, 14E30. 
2 OSAMU FUJINO, HIROSHI SATO, YUKISHIGE TAKANO, HOKUTO UEHARA

Acknowledgments. We would like to thank Professor Kenji Matsuki, who informed us that Professor Yujiro Kawamata pointed out an error in [M, Remark 14-2-7]. The first author was partially supported by the Grant-in-Aid for Young Scientists (A) $\sharp 17684001$ from JSPS. He was also supported by the Inamori Foundation. The fourth author was partially supported by the Grant-in-Aid for Young Scientists (B) $\sharp 17740012$ from JSPS.

Notation. We will work over $\mathbb{C}$, the complex number field, throughout this paper. Let $v_{i} \in N \simeq \mathbb{Z}^{3}$ for $1 \leq i \leq k$. Then the symbol $\left\langle v_{1}, v_{2}, \cdots, v_{k}\right\rangle$ denotes the cone $\mathbb{R}_{\geq 0} v_{1}+\mathbb{R}_{\geq 0} v_{2}+\cdots+\mathbb{R}_{\geq 0} v_{k}$ in $N_{\mathbb{R}} \simeq$ $N \otimes_{\mathbb{Z}} \mathbb{R}$

\section{ThreE-Dimensional TERMinAl TORIC Singularities}

In this section, we characterize non- $\mathbb{Q}$-factorial affine toric threefolds with terminal singularities. We will use the same notation as in [YPG], which is an excellent exposition on terminal singularities.

Let $X$ be an affine toric threefold. First, let us recall the following well-known theorem of G. K. White, D. Morrison, G. Stevens, V. Danilov, and M. Frumkin (see [YPG, (5.2) Theorem]).

Theorem 2.1. Assume that $X$ is $\mathbb{Q}$-factorial. Then $X$ is terminal if and only if (up to permutations of $(x, y, z)$ and symmetries of $\mu_{r}$ ) $X \simeq \mathbb{C}^{3} / \mu_{r}$ of type $\frac{1}{r}(a,-a, 1)$ with a coprime to $r$, where $\mu_{r}$ is the cyclic group of order $r$. In particular, if $X$ is Gorenstein and terminal, then $X$ is non-singular.

Here, we prove the following well-known result for the reader's convenience (cf. [I], and [II, Theorem 3.6]).

Theorem 2.2. Assume that $X$ is not $\mathbb{Q}$-factorial. Then $X$ is terminal if and only if $X \simeq \operatorname{Spec} \mathbb{C}[x, y, z, w] /(x y-z w)$. We call this singularity an ordinary double point.

By the above theorems, we obtain the complete list of three-dimensional terminal toric singularities.

Remark 2.3. Mori classified three-dimensional terminal singularities. For the details, see [YPG, (6.1) Theorem]. We do not use his classification table in this paper.

Proof of Theorem 2.2. Let $N=\mathbb{Z}^{3}$ and $\Delta=\left\langle e_{1}, \cdots, e_{k}\right\rangle$ the cone in $N$ such that $X=X(\Delta)$, where each $e_{i}$ is primitive. First, we prove

Claim 1. If $X$ is non- $\mathbb{Q}$-factorial and terminal, then $k=4$. 
Proof of the claim. It is obvious that $k \geq 4$. Since $X$ is $\mathbb{Q}$-Gorenstein, there is a hyperplane $H \subset N$ that contains every $e_{i}$. On $H \simeq \mathbb{Z}^{2}$, $e_{i}$ s span two dimensional convex polygon $P$. By renumbering $e_{i}$ s, we can assume that they are arranged counter-clockwise. Since $X(\Delta)$ is terminal, all the lattice points in $P$ are $e_{i}$ s. In particular, the triangle on $H$ spanned by $e_{1}, e_{2}$, and $e_{3}$ contains only three lattice points $e_{i}$ $(1 \leq i \leq 3)$ of $H$. So, after changing the coordinate of $H$, we can assume that $e_{1}=(0,1), e_{2}=(0,0)$, and $e_{3}=(1,0)$ in $H \simeq \mathbb{Z}^{2}$. It can be checked easily that $(1,1) \in P$ since $k \geq 4$. Thus, we obtain that $k=4$ and $e_{4}=(1,1)$.

Claim 2. Assume that $X$ is non- $\mathbb{Q}$-factorial, Gorenstein, and terminal. Then $X$ is isomorphic to $\operatorname{Spec} \mathbb{C}[x, y, z, w] /(x y-z w)$.

Proof of the claim. On this assumption, the cones $\left\langle e_{1}, e_{2}, e_{3}\right\rangle,\left\langle e_{1}, e_{2}, e_{4}\right\rangle$, $\left\langle e_{1}, e_{3}, e_{4}\right\rangle$, and $\left\langle e_{2}, e_{3}, e_{4}\right\rangle$ define $\mathbb{Q}$-factorial Gorenstein affine toric threefolds with terminal singularities. By Theorem 2.1, every cone listed above is non-singular. So, by changing the coordinate of $N$, we can assume that $e_{1}=(1,0,0), e_{2}=(0,1,0)$, and $e_{3}=(0,0,1)$. Since $X$ is Gorenstein and $\left\langle e_{1}, e_{2}, e_{4}\right\rangle,\left\langle e_{1}, e_{3}, e_{4}\right\rangle$, and $\left\langle e_{2}, e_{3}, e_{4}\right\rangle$ are non-singular, $e_{4}=(-1,1,1),(1,-1,1)$, or $(1,1,-1)$. Anyway, we can check that $X \simeq \operatorname{Spec} \mathbb{C}[x, y, z, w] /(x y-z w)$.

By the above claim, it is sufficient to prove

Claim 3. All the non-Q- $\mathbb{Q}$-factorial toric affine threefolds with terminal singularities are Gorenstein.

Proof of the claim. We assume that $X$ is not Gorenstein and obtain a contradiction.

Let $\bar{N}$ be the sublattice of $N$ spanned by all the lattice points on $H$ and the origin of $N$. In $\bar{N}, \Delta=\left\langle e_{1}, e_{2}, e_{3}, e_{4}\right\rangle$ defines a Gorenstein terminal threefold. So, we can assume that $e_{1}=(1,0,0), e_{2}=(0,1,0)$, $e_{3}=(0,0,1)$, and $e_{4}=(1,1,-1) \in \mathbb{Z}^{3} \simeq \bar{N}$ by the proof of Claim 2. First, we consider $\left\langle e_{1}, e_{2}, e_{3}\right\rangle$ in $\bar{N}$ and $N$. By Theorem 2.1, we obtain that $N=\bar{N}+\mathbb{Z} \cdot \frac{1}{r}(\alpha, \beta, \gamma)$, where $(\alpha, \beta, \gamma)$ is one of the followings: $(a,-a, 1),(a, 1,-a),(-a, a, 1),(-a, 1, a),(1, a,-a),(1,-a, a)$ such that $0<a<r$ with $a$ coprime to $r$. Next, we use the terminality of $\left\langle e_{1}, e_{2}, e_{4}\right\rangle$. We consider the linear transform $T: N \rightarrow N$ such that $T e_{1}=e_{1}, T e_{2}=e_{2}, T e_{4}=e_{3}$. Then $T N=T \bar{N}+\mathbb{Z} \cdot \frac{1}{r}\left(\alpha^{\prime}, \beta^{\prime}, \gamma^{\prime}\right)$, where $\left(\alpha^{\prime}, \beta^{\prime}, \gamma^{\prime}\right)$ is one of the followings: $(1+a, 1-a,-1),(0,1-a, a)$, 
4 OSAMU FUJINO, HIROSHI SATO, YUKISHIGE TAKANO, HOKUTO UEHARA

$(1-a, 1+a,-1),(0,1+a,-a),(1-a, 0, a),(1+a, 0,-a)$. Note that

$$
\left(\begin{array}{l}
\alpha^{\prime} \\
\beta^{\prime} \\
\gamma^{\prime}
\end{array}\right)=\left(\begin{array}{ccc}
1 & 0 & 1 \\
0 & 1 & 1 \\
0 & 0 & -1
\end{array}\right)\left(\begin{array}{l}
\alpha \\
\beta \\
\gamma
\end{array}\right) \text {. }
$$

We treat the first case, that is, $\left(\alpha^{\prime}, \beta^{\prime}, \gamma^{\prime}\right)=(1+a, 1-a,-1)$. By the terminal lemma (see [YPG, (5.4) Theorem]), $r$ divides $(1+a)+(1-a)=$ 2 since it does not divide $(1+a)+(-1)$ nor $(1-a)+(-1)$. So, $r=2$ and $a=1$. Thus $\frac{1}{r}\left(\alpha^{\prime}, \beta^{\prime}, \gamma^{\prime}\right)=\frac{1}{2}(2,0,-1) \equiv \frac{1}{2}(0,0,1)(\bmod T \bar{N})$. It is a contradiction (see Theorem 2.1). We leave the other cases for the reader's exercise. So, there are no non-Gorenstein non- $\mathbb{Q}$-factorial affine toric threefolds with terminal singularities.

Therefore, we completed the proof of Theorem 2.2.

Theorem 2.2 has a beautiful corollary.

Corollary 2.4 (Three-dimensional terminal toric flop). Let

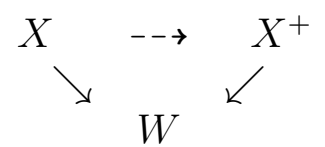

be a three-dimensional toric flopping diagram such that $W$ is affine. Assume that $X$ has only terminal singularities. Then it is the simplest flop, where the simplest flop means the flop described in [F1, p.49-p.50].

Proof. By the assumption, $W$ is a non- $\mathbb{Q}$-factorial affine toric threefold with terminal singularities. Thus, $X \simeq \operatorname{Spec} \mathbb{C}[x, y, z, w] /(x y-z w)$ by Theorem 2.2. So, the above diagram must be the simplest flop.

\section{Three-DIMEnsional $\mathbb{Q}$-FACTORIAL TERMINAL TORIC FLIPS}

We classify three-dimensional flipping contractions from $\mathbb{Q}$-factorial terminal toric threefolds. The next theorem was stated in [KMM] without proof at the end of Example 5-2-5.

Theorem 3.1 (Three-dimensional $\mathbb{Q}$-factorial terminal toric flips). Let $\varphi_{R}: X(\Delta) \rightarrow Y(\Sigma)$ be the contraction morphism of an extremal ray $R$ with $K_{X} \cdot R<0$ of flipping type from a toric threefold with only $\mathbb{Q}$-factorial terminal singularities. Assume that $Y$ is affine. Then we have the following description of the flipping contraction:

There exist two three-dimensional cones

$$
\begin{aligned}
& \tau_{4}=\left\langle v_{1}, v_{2}, v_{3}\right\rangle \in \Delta, \\
& \tau_{3}=\left\langle v_{1}, v_{2}, v_{4}\right\rangle \in \Delta,
\end{aligned}
$$


sharing the two-dimensional wall

$$
w=\left\langle v_{1}, v_{2}\right\rangle
$$

such that $[V(w)] \in R$ and that for some $\mathbb{Z}$-coordinate of $N \simeq \mathbb{Z}^{3}$,

$$
\begin{aligned}
& v_{1}=(1,0,0), \\
& v_{4}=(a, r-a,-r),
\end{aligned} \quad v_{2}=(0,1,0), \quad v_{3}=(0,0,1),
$$

or

$$
\begin{aligned}
& v_{1}=(1,0,0), \quad v_{2}=(0,1,0), \quad v_{3}=(0,0,1), \\
& v_{4}=(a, 1,-r),
\end{aligned}
$$

where $0<a<r$ and $\operatorname{gcd}(r, a)=1$. Therefore,

$$
\Delta=\left\{\tau_{3}, \tau_{4}, \text { and their faces }\right\}
$$

and

$$
\Sigma=\left\{\left\langle v_{1}, v_{2}, v_{3}, v_{4}\right\rangle \text {, and its faces }\right\} .
$$

Proof. By [M, Example-Claim 14-2-5], it is sufficient to prove that the (unique) rational curve that is contracted passes through only one singular point of $X$. Without loss of generality, we may assume that $v_{1}=(1,0,0)$ and $v_{2}=(0,1,0)$ since $\left\langle v_{1}, v_{2}\right\rangle$ is a two-dimensional nonsingular cone. Seeking a contradiction, we assume that both $\left\langle v_{1}, v_{2}, v_{3}\right\rangle$ and $\left\langle v_{1}, v_{2}, v_{4}\right\rangle$ are singular. By the terminal lemma ([O, $\left.\left.\S 1.6\right]\right)$, we may assume that $v_{3}=(1, p, q)$, where $0<p<q$ and $\operatorname{gcd}(p, q)=1$. We note that $q \geq 2$. We can write $v_{4}=a v_{1}+b v_{2}+c(k, l,-1)$ with $0<a<c$, $0<b<c, \operatorname{gcd}(a, c)=1, \operatorname{gcd}(b, c)=1$, and $k, l \in \mathbb{Z}$. In particular, $c \geq 2$. We note that we assumed that $\left\langle v_{1}, v_{2}, v_{4}\right\rangle$ is singular and terminal. By the terminal lemma again (see [O, p.36 White's Theorem]), at least one of $a-1, b-1$ and $a+b$ is divisible by $c$. Therefore, $a=1$, $b=1$, or $a+b=c$. We note that $v_{1}, v_{2}, v_{3}$ are on the plane

$$
x+y-\frac{p}{q} z=1
$$

Case $1(a=1)$. In this case, $v_{4}=(1+c k, b+c l,-c)$. We have

$$
\frac{c}{q} v_{3}+v_{4}=\left(1+c k+\frac{c}{q}, b+c l+\frac{p}{q} c, 0\right) .
$$

Thus, we obtain the following three inequalities:

$$
\begin{aligned}
& 1+c k+\frac{c}{q}>0, \\
& b+c l+\frac{p}{q} c>0,
\end{aligned}
$$


6 OSAMU FUJINO, HIROSHI SATO, YUKISHIGE TAKANO, HOKUTO UEHARA

and

$$
1+c k+b+c l+\frac{p}{q} c<1
$$

The inequalities (11) and (2) follow from the condition that $\varphi_{R}$ is small. The condition $K_{X} \cdot R<0$ implies the inequality (3). By (2) and (3), we have $k \leq-1$. Thus

$$
0<1+c k+\frac{c}{q} \leq 1-c+\frac{c}{q} \leq 1-\frac{1}{2} c \leq 0
$$

by (1). It is a contradiction.

Case $2(b=1)$. In this case, $v_{4}=(a+c k, 1+c l,-c)$. We have

$$
\frac{c}{q} v_{3}+v_{4}=\left(a+c k+\frac{c}{q}, 1+c l+\frac{p}{q} c, 0\right) .
$$

Thus, we obtain the following three inequalities:

$$
\begin{aligned}
& a+c k+\frac{c}{q}>0, \\
& 1+c l+\frac{p}{q} c>0,
\end{aligned}
$$

and

$$
a+c k+1+c l+\frac{p}{q} c<1 .
$$

By (15) and (6),$k \leq-1$. So, $k=-1$ by (41). By (5), we know that $l \geq-1$. Therefore, $l=0$ or -1 by (마).

First, we assume that $l=0$. Then we get

$$
a-c+\frac{p}{q} c<0
$$

by (6) and

$$
a-c+\frac{c}{q}>0
$$

by (41). It is a contradiction.

Next, we assume that $l=-1$. Then we obtain

$$
a-c+\frac{c}{q}>0
$$

by (4) and

$$
1-c+\frac{p}{q} c>0
$$

by (5). These two inequalities imply that

$$
1+a-2 c+\frac{p+1}{q} c>0 .
$$


It is a contradiction.

Case $3(a+b=c)$. In this case, $v_{4}=(a+c k, c-a+c l,-c)$. We have

$$
\frac{c}{q} v_{3}+v_{4}=\left(a+c k+\frac{c}{q}, c-a+c l+\frac{p}{q} c, 0\right) .
$$

Thus, we obtain the following three inequalities:

$$
\begin{gathered}
a+c k+\frac{c}{q}>0, \\
c-a+c l+\frac{p}{q} c>0,
\end{gathered}
$$

and

$$
a+c k+c-a+c l+\frac{p}{q} c<1 .
$$

By (8) and (9), $k \leq-1$. So, $k=-1$ by (7). By (8), we have $l \geq-1$. Therefore, $l=0$ or -1 by (9).

First, we assume that $l=0$. Then we have

$$
\frac{p}{q} c<1
$$

by (9) and

$$
a-c+\frac{c}{q}>0
$$

by (7). Thus,

$$
1>\frac{p}{q} c \geq \frac{c}{q}>c-a \geq 1
$$

It is a contradiction.

Next, we assume that $l=-1$. Then we obtain

$$
a-c+\frac{c}{q}>0
$$

by (7) and

$$
-a+\frac{p}{q} c>0
$$

by (8). By adding these two inequalities, we have

$$
-c+\frac{p+1}{q} c>0 \text {. }
$$

It is a contradiction.

Therefore, at least one of $\left\langle v_{1}, v_{2}, v_{3}\right\rangle$ and $\left\langle v_{1}, v_{2}, v_{4}\right\rangle$ must be nonsingular. Thus, we have the desired description of $\varphi_{R}: X \rightarrow Y$ by [M], Example-Claim 14-2-5]. 
8 OSAMU FUJINO, HIROSHI SATO, YUKISHIGE TAKANO, HOKUTO UEHARA

Remark 3.2. The example in [M, Remark 14-2-7 (ii)] is not true. The cone $\left\langle v_{1}, v_{2}, v_{3}\right\rangle$ is not terminal. The cone $\left\langle v_{1}, v_{2}, v_{3}\right\rangle$ has canonical singularities.

Remark 3.3. The source space $X$ in Theorem 3.1 is always singular.

Remark 3.4. In [M, Example-Claim 14-2-5], $X$ is assumed to be complete. It is because contraction morphisms of extremal rays are constructed only for complete varieties in [R] and [M, Chapter 14]. For the details of non-complete toric varieties, see [FS], [F1], and [S].

\section{Main Theorem}

The following theorem is the main theorem of this paper.

Theorem 4.1 (cf. [T]). Let $\varphi: X \rightarrow Y$ be a small proper toric morphism such that $X$ is a three-dimensional toric variety with only terminal singularities. Let $C \simeq \mathbb{P}^{1}$ be an exceptional curve of $\varphi$. Assume that $-K_{X} \cdot C>0$. Then $C$ does not pass through ordinary double points.

Proof. First, we assume that $C$ passes through two ordinary double points. By taking a small projective resolution of $X$, we can assume that $C$ does not pass through any singular points. It is a contradiction by Theorem 3.1 (see Remark 3.3).

Next, we assume that $C$ passes through only one ordinary double points. By Theorem 3.1, we have the following local description of $X$ and $C$ :

There exist lattice points of $N=\mathbb{Z}^{3}$

$$
\begin{aligned}
& v_{1}=(1,0,0), \quad v_{2}=(0,1,0), \quad v_{3}=(0,0,1), \\
& v_{5}=(-1,1,1), \quad v_{6}=(1,-1,1) .
\end{aligned}
$$

We put

$$
\Delta_{1}=\left\{\left\langle v_{1}, v_{2}, v_{3}, v_{5}\right\rangle,\left\langle v_{1}, v_{2}, v_{4}\right\rangle, \text { and their faces }\right\}
$$

and

$$
\Delta_{2}=\left\{\left\langle v_{1}, v_{2}, v_{3}, v_{6}\right\rangle,\left\langle v_{1}, v_{2}, v_{4}\right\rangle, \text { and their faces }\right\},
$$

where $v_{4}=(a, r-a,-r)$ or $(a, 1,-r)$ with $0<a<r$ and $\operatorname{gcd}(a, r)=1$. Then $X=X(\Delta)$, where $\Delta=\Delta_{1}$ or $\Delta_{2}$, and $C$ is $V\left(\left\langle v_{1}, v_{2}\right\rangle\right) \simeq \mathbb{P}^{1}$.

Case 1. When $v_{4}=(a, r-a,-r)$ and $\Delta=\Delta_{1}$, we have

$$
v_{2}=\frac{r}{2 r-a} v_{5}+\frac{r-a}{2 r-a} v_{1}+\frac{1}{2 r-a} v_{4} \text {. }
$$

Therefore, $v_{2}$ is contained in the cone $\left\langle v_{5}, v_{1}, v_{4}\right\rangle$. Thus, we can not remove the wall $\left\langle v_{1}, v_{2}\right\rangle$ from $\Delta$. 
Case 2. When $v_{4}=(a, 1,-r)$ and $\Delta=\Delta_{1}$, we have

$$
v_{2}=\frac{r}{r+1} v_{5}+\frac{r-a}{r+1} v_{1}+\frac{1}{r+1} v_{4} \text {. }
$$

Therefore, $v_{2}$ is contained in the cone $\left\langle v_{5}, v_{1}, v_{4}\right\rangle$. Thus, we can not remove the wall $\left\langle v_{1}, v_{2}\right\rangle$ from $\Delta$.

Case 3. When $v_{4}=(a, r-a,-r)$ and $\Delta=\Delta_{2}$, we have

$$
v_{1}=\frac{r}{r+a} v_{6}+\frac{a}{r+a} v_{2}+\frac{1}{r+a} v_{4} .
$$

Therefore, $v_{1}$ is contained in the cone $\left\langle v_{6}, v_{2}, v_{4}\right\rangle$. Thus, we can not remove the wall $\left\langle v_{1}, v_{2}\right\rangle$ from $\Delta$.

Case 4. When $v_{4}=(a, 1,-r)$ and $\Delta=\Delta_{2}$, we have

$$
v_{1}=\frac{r}{r+a} v_{6}+\frac{r-1}{r+a} v_{2}+\frac{1}{r+a} v_{4} .
$$

Therefore, $v_{1}$ is contained in the cone $\left\langle v_{6}, v_{2}, v_{4}\right\rangle$. Thus, we can not remove the wall $\left\langle v_{1}, v_{2}\right\rangle$ from $\Delta$.

Thus, $C$ does not pass through any ordinary double points.

\section{REFERENCES}

[F1] O. Fujino, Equivariant completions of toric contraction morphisms, Tohoku Math. J. 58 (2006), 303-321.

[F2] O. Fujino, Special termination and reduction to pl flips, 63-75 in Flips for 3-folds and 4-folds, Oxford University Press (2007).

[FS] O. Fujino and H. Sato, Introduction to the toric Mori theory, Michigan Math. J. 52 (2004), no. 3, 649-665.

[Fl] W. Fulton, Introduction to toric varieties, Annals of Mathematics Studies, 131, The William H. Roever Lectures in Geometry, Princeton University Press, Princeton, NJ, 1993.

[I] M. Ishida, On the terminal toric singularities of dimension three, in Commutative Algebra, Karuizawa, Japan, 1982 (S. Goto, ed.), 54-70.

[II] M. Ishida, and N. Iwashita, Canonical cyclic quotient singularities of dimension three, Complex analytic singularities, 135-151, Adv. Stud. Pure Math., 8, North-Holland, Amsterdam, 1987.

[KMM] Y. Kawamata, K. Matsuda, and K. Matsuki, Introduction to the minimal model problem, Algebraic geometry, Sendai, 1985, 283-360, Adv. Stud. Pure Math., 10, North-Holland, Amsterdam, 1987.

[M] K. Matsuki, Introduction to the Mori program, Universitext, SpringerVerlag, New York, 2002.

[O] T. Oda, Convex bodies and algebraic geometry. An introduction to the theory of toric varieties, Translated from the Japanese. Ergebnisse der Mathematik und ihrer Grenzgebiete (3) [Results in Mathematics and Related Areas (3)], 15. Springer-Verlag, Berlin, 1988. viii+212 pp. 
10OSAMU FUJINO, HIROSHI SATO, YUKISHIGE TAKANO, HOKUTO UEHARA

[R] M. Reid, Decomposition of toric morphisms, Arithmetic and geometry, Vol. II, 395-418, Progr. Math. 36, Birkhäuser Boston, Boston, MA, 1983.

[YPG] M. Reid, Young person's guide to canonical singularities, Algebraic geometry, Bowdoin, 1985 (Brunswick, Maine, 1985), 345-414, Proc. Sympos. Pure Math., 46, Part 1, Amer. Math. Soc., Providence, RI, 1987.

[S] H. Sato, Combinatorial descriptions of toric extremal contractions, Nagoya Math. J. 180 (2005), 111-120.

[T] Y. Takano, On flipping contractions of three-dimensional toric varieties with non- $\mathbb{Q}$-factorial terminal singularities (Japanese), Master's thesis, Tokyo Metropolitan University (2008).

Graduate School of Mathematics, Nagoya University, Chikusa-Ku NAGOYA 464-8602 JAPAN

E-mail address: fujino@math.nagoya-u.ac.jp

Faculty of Economics and Information, Gifu Shotoku Gakuen UniVERSITY, 1-38 NAKAUZURA GIFU 500-8288 JAPAN

E-mail address: hirosato@gifu.shotoku.ac.jp

Department of Mathematics, Tokyo Metropolitan University, 1-1 Minami-Ohsawa HachioJi-Shi TOKYo 192-0397 Japan

Department of Mathematics, Tokyo Metropolitan University, 1-1 Minami-OhsaWa HachioJi-Shi ToKyo 192-0397 Japan

E-mail address: hokuto@tmu.ac.jp 